\title{
Uma Experiência no Ensino de Informática para Deficientes Visuais no Município de Garanhuns-PE
}

\author{
Aline Ferreira Barbosa ${ }^{1}$, Roseane de O. Martins', Higor Ricardo M. Santos ${ }^{2}$ \\ ${ }^{1}$ Universidade de Pernambuco, Campus Garanhuns (UPE) \\ CEP 55.294-902 - Garanhuns - PE - Brasil \\ ${ }^{2}$ Centro de Informática - Universidade Federal de Pernambuco (UFPE) \\ CEP 50.740-560 - Recife - PE - Brasil \\ \{alineferreirabarbosa@gmail.com, roseanegus@gmail.com, \\ higormonteiro1206@gmail.com\}
}

\begin{abstract}
The purpose of this paper is to present an experience report on a training course in Assistive Technologies for the visually impaired in the municipality of Garanhuns-PE. The motivation of this research concerns the direction of literature on digital inclusion and improving the use of technology by the visually impaired. This study had the opportunity to offer participants a training in the use of Dosvox and NVDA software to access the basic features of the computer, including access to the Web. According to participants, the project conducted promoted a change in perspective about the importance of computer use. It is expected that other courses Computer Science Teaching repeated and refine the practices presented in this paper.
\end{abstract}

Resumo. O objetivo deste artigo é apresentar um relato de experiência acerca de um curso de formação em Tecnologias Assistivas para deficientes visuais do município de Garanhuns-PE. A motivação desta pesquisa aborda o direcionamento da literatura a respeito da inclusão digital e melhoria da utilização das tecnologias por parte dos deficientes visuais. Este trabalho teve a oportunidade de oferecer aos participantes uma capacitação no uso dos softwares Dosvox e NVDA para acessar os recursos básicos do computador, inclusive o acesso à Web. De acordo com os participantes, o projeto realizado promoveu uma mudança de perspectiva sobre a importância do uso do computador. Espera-se que outros cursos de Licenciatura em Computação repitam e aperfeiçoem as práticas apresentadas neste trabalho.

\section{Introdução}

Devido à imersão das Tecnologias de Informação e Comunicação (TIC) na vida cotidiana das pessoas, tem-se percebido uma mudança social na forma de interação entre elas, novos métodos de trabalho, aumento dos meios de acesso à informação e até no modo de se entreter. No entanto, ainda existe uma lacuna grande quando pessoas com algum tipo de deficiência querem se inserir nesse contexto. Seja por falta de tecnologias apropriadas ou por não saberem utilizar tais tecnologias. Nesse aspecto, $\mathrm{o}$ conceito de Tecnologias Assistivas é aplicado como um meio de minimizar as barreiras atreladas à deficiência e promover a inclusão digital e social desses indivíduos.

De acordo com o censo demográfico do IBGE de 2010, cerca de um quarto da população brasileira tem pelo menos um tipo de deficiência, seja ela visual, auditiva, motora ou intelectual, totalizando cerca de 45 milhões de pessoas. $\mathrm{O}$ número equivale a 
24\% dos 190 milhões de habitantes do País. A deficiência visual foi a mais citada, detectou-se que 18,8\% dos brasileiros têm dificuldade para enxergar ou possui cegueira total. Diante de dados tão significativos de pessoas que possuem deficiência visual, percebe-se a necessidade de realizar cursos de formação voltados para esses indivíduos com o intuito de prepará-los no uso de Tecnologias Assistivas.

Neste contexto, diversos fatores apontam a relevância do ensino de Informática para deficientes visuais por meio das Tecnologias Assistivas. Primeiramente, este tipo de educação aliada ao uso do computador facilita a comunicação no meio digital, assim promovendo uma inclusão social e tecnológica. Além disso, o uso efetivo do computador permite contribuir para um avanço qualitativo do processo de ensino e aprendizagem, como também para o resgate da autoestima dos alunos com deficiência visual.

A Educação Inclusiva constitui um novo modelo de educação em que é possível o acesso e a permanência de todos os alunos, onde são utilizados procedimentos de identificação e remoção de barreiras para a aprendizagem (GLAT, 2007). Conforme Borges (2012), "uma pessoa cega pode ter algumas limitações, as quais poderão trazer obstáculos ao seu aproveitamento produtivo na sociedade". Ele aponta que grande parte destas limitações pode ser eliminada através de duas ações: uma educação adaptada à realidade destes sujeitos e o uso da tecnologia para diminuir essas barreiras.

Nesse aspecto, se faz presente o uso das Tecnologias Assistivas, também chamadas de tecnologia adaptativa ou tecnologia de apoio. Tecnologias Assistivas referem-se a qualquer ferramenta ou recurso destinado a proporcionar à pessoa portadora de deficiência maior independência, qualidade de vida e mobilidade (FERREIRA; NUNES, 2011). Um exemplo dessa tecnologia são os softwares sintetizadores de voz, os quais permitem ao deficiente visual utilizar o computador.

No intuito de fundamentar o presente trabalho, buscou-se na literatura relatos de experiências que obtiveram sucesso em suas realizações. Santos et al. (2012) relata um curso de formação para deficientes visuais numa cidade distante dos grandes centros do Rio Grande do Sul. Em seu trabalho, foi percebido que o curso estimulou atitudes e desenvolveu habilidades que os tornaram mais capazes para a participação social cidadã e para a vida produtiva. Pansanato et al. (2012) descreve a experiência de inclusão de um estudante cego na educação superior em computação. Ao longo da graduação, os professores tiveram que se adaptar e desenvolver, juntamente com um facilitador, mecanismos para facilitar a interpretação dos diagramas Entidade-Relacionamento, Autômatos, entre outros. Souza (2004) realizou um estudo de caso para observar e analisar o processo de apropriação das TIC's pelos deficientes visuais. Ela utilizou o Dosvox para as interações com os participantes, por se tratar de um programa simples, intuitivo, gratuito, de fácil instalação e em português.

Nesse sentido, este trabalho foi desenvolvido a partir de duas principais motivações. Primeiramente, detectou-se em pesquisas anteriores um direcionamento para a inclusão digital e melhoria da utilização das tecnologias por parte dos deficientes visuais. Em seguida, de acordo com a Associação dos Deficientes Visuais do Agreste Meridional de Pernambuco (ADVAMPE), situada no município de Garanhuns, distante $228 \mathrm{~km}$ da capital Recife/PE, os deficientes visuais deste município não possuem formação tecnológica adaptada às suas necessidades. 
Este artigo apresenta as atividades desenvolvidas durante um projeto de extensão do curso de Licenciatura em Computação da Universidade de Pernambuco. O objetivo principal foi capacitar deficientes visuais para utilizar o computador como ferramenta produtiva diária através de Tecnologias Assistivas. Para isso, foram utilizados os softwares Dosvox ${ }^{1}$ e Non Visual Desktop Access - NVDA $^{2}$, uma vez que estes desempenham um papel fundamental no cotidiano das pessoas com deficiência visual, ampliam as possibilidades de comunicação e autonomia pessoal. Além disso, eles atuam minimizando ou compensando as restrições procedentes da ausência da visão.

As contribuições vislumbradas a partir desta experiência abrangem tanto o meio social em que a universidade está inserida, como também para a educação dos graduandos em Licenciatura em Computação com a promoção de práticas inclusivas voltadas para o ensino de informática. Com a divulgação, discussão e replicação deste relato de experiência, desenvolve-se a oportunidade de ampliar o interesse e aperfeiçoar os métodos de ensino e aprendizagem para atender as necessidades dos deficientes visuais. Com isso, será possível potencializar a inserção deles no mercado de trabalho e nas Universidades.

\section{Experiência da utilização de Tecnologias Assistivas para deficientes visuais}

\subsection{Planejamento e componente curricular do curso de formação}

Este projeto contou com a orientação de um professor de graduação e duas alunas de Licenciatura em Computação que atuaram como tutoras do curso de formação dos deficientes visuais. O planejamento deste projeto foi dividido em duas etapas: a reflexão-ação no desenvolvimento de metodologias para o ensino e aprendizagem de pessoas com deficiência e a formação de deficientes visuais para utilização de tecnologias assistivas.

A primeira etapa consistiu na pesquisa e elaboração de estratégias de ensino e aprendizagem de tecnologia para pessoas portadoras de deficiência com base em conceitos relacionados à Educação Inclusiva. A metodologia para esta atividade tem como proposta curricular: a) Leitura e discussão sobre as Tecnologias Assistivas como parte da Educação Inclusiva; b) Implementação de estratégias que permitam auxiliar os alunos com deficiência na comunicação e nas suas atividades dentro e fora dos espaços escolares.

A segunda etapa contemplou a obtenção do público-alvo junto a ADVAMPE, em que foram selecionados oito deficientes visuais associados. Além disso, participou também uma pessoa não portadora de deficiência visual com a intenção de posteriormente ser a responsável por transmitir o conhecimento a outros portadores de deficiência visual desta associação.

O curso de formação foi realizado durante quatro meses em um laboratório de informática do Telecentro de Garanhuns. Telecentro é um espaço público onde pessoas

\footnotetext{
${ }^{1}$ É um sistema para microcomputadores da linha PC que se comunica com o usuário através de síntese de voz, viabilizando, deste modo, o uso de computadores por deficientes visuais, que adquirem assim, um alto grau de independência no estudo e no trabalho (DOSVOX, 2012).

${ }^{2}$ NVDA é um leitor de tela que se comunica com o usuário através de síntese de voz, sua tradução em português é Acesso Não-Visual ao Ambiente de Trabalho (NVDA, 2012).
} 
podem utilizar microcomputadores, a Internet e outras tecnologias digitais que permitem coletar informações, criar, aprender e comunicar-se com outras pessoas, enquanto desenvolvem habilidades digitais essenciais do Século 21. Com uma carga horária de 2 horas semanais, a estrutura das aulas foi composta por dois momentos: primeiramente eram ministradas aulas expositivas e posteriormente realizaram-se atividades práticas utilizando os softwares Dosvox e NVDA. O projeto contou com o apoio da UPE, Pró-reitora de Extensão e Cultura, Secretaria de Desenvolvimento Econômico deste município e da TEC $\mathrm{Jr}^{3}$.

\subsection{Caracterização dos participantes}

No início do curso, buscou-se caracterizar o perfil dos participantes. Além do tipo de deficiência, sexo e recurso tecnológico que eles possuíam, realizou-se um levantamento do nível de conhecimento e experiência em informática dos participantes com relação ao uso de Tecnologias Assistivas. A Tabela 1 demonstra o perfil de todos os participantes. Seus respectivos nomes foram omitidos por questões de privacidade.

Tabela 1. Perfil dos participantes

\begin{tabular}{|c|c|c|c|c|}
\hline Participante & $\begin{array}{c}\text { Tipo de } \\
\text { deficiência }\end{array}$ & Sexo & $\begin{array}{l}\text { Experiência em } \\
\text { Informática }\end{array}$ & $\begin{array}{c}\text { Recurso } \\
\text { tecnológico }\end{array}$ \\
\hline 1 & Cegueira total & Feminino & $\begin{array}{c}\text { Não utiliza o Dosvox } \\
\text { e o NVDA }\end{array}$ & $\begin{array}{c}\text { Possui } \\
\text { Computador } \\
\text { pessoal. }\end{array}$ \\
\hline 2 & Cegueira total & Masculino & $\begin{array}{c}\text { Não utiliza o Dosvox } \\
\text { e o NVDA }\end{array}$ & $\begin{array}{c}\text { Não possui } \\
\text { computador em } \\
\text { casa. }\end{array}$ \\
\hline 3 & Cegueira total & Feminino & $\begin{array}{c}\text { Utiliza o Dosvox e } \\
\text { não utilizava o } \\
\text { NVDA }\end{array}$ & Possui notebook \\
\hline 4 & Baixa visão & Feminino & $\begin{array}{c}\text { Não utiliza o Dosvox } \\
\text { e o NVDA }\end{array}$ & $\begin{array}{l}\text { Possui } \\
\text { computador } \\
\text { pessoal. }\end{array}$ \\
\hline 5 & Cegueira total & Feminino & $\begin{array}{c}\text { Não utiliza o Dosvox } \\
\text { e o NVDA }\end{array}$ & $\begin{array}{l}\text { Não possui } \\
\text { computador } \\
\text { pessoal. }\end{array}$ \\
\hline 6 & Cegueira total & Masculino & $\begin{array}{c}\text { Não utiliza o Dosvox } \\
\text { e o NVDA }\end{array}$ & $\begin{array}{l}\text { Possui } \\
\text { computador } \\
\text { pessoal. }\end{array}$ \\
\hline
\end{tabular}

\footnotetext{
${ }^{3}$ TEC JR (Tecnologia, Educação e Consultoria Júnior) é a primeira empresa júnior brasileira de um curso de Licenciatura em Computação. Entre seus serviços oferecidos, os principais são desenvolvimento de softwares educacionais e capacitações na área de computação http://tecjr.com/sobre.html.
} 


\begin{tabular}{|c|l|l|l|c|}
\hline 7 & Cegueira total & Feminino & $\begin{array}{c}\text { Não utiliza o Dosvox } \\
\text { e o NVDA }\end{array}$ & $\begin{array}{c}\text { Não possui } \\
\text { computador } \\
\text { pessoal. }\end{array}$ \\
\hline 8 & Baixa visão & Masculino & $\begin{array}{c}\text { Utiliza o Dosvox e } \\
\text { não utiliza o NVDA }\end{array}$ & $\begin{array}{c}\text { Possui } \\
\text { computador } \\
\text { pessoal. }\end{array}$ \\
\hline 9 & $\begin{array}{c}\text { Não possui } \\
\text { deficiência }\end{array}$ & Feminino & $\begin{array}{c}\text { Não utiliza o Dosvox } \\
\text { e o NVDA }\end{array}$ & $\begin{array}{c}\text { Possui } \\
\text { computador } \\
\text { pessoal. }\end{array}$ \\
\hline
\end{tabular}

Ao analisar a Tabela 1, observa-se que apenas 2 alunos utilizavam o Dosvox e nenhum dos participantes tinha experiência com o NVDA previamente. Fato que comprovou a informação da ADVAMPE sobre a pouca utilização das tecnologias assistivas pelos deficientes visuais. A partir disso, torna-se necessário motivar e justificar a esse público sobre os diversos benefícios que esses softwares podem desempenhar para sua independência nas tarefas diárias que envolvam o uso do computador.

\subsection{Ações Desenvolvidas}

As ações desenvolvidas estão categorizadas em três etapas: conceitos introdutórios sobre informática (Sistema Computacional, Hardware, Software, Periféricos), ensino do Dosvox e o ensino do NVDA. Por apresentar algumas características diferentes, os conteúdos de ensino do Dosvox e do NVDA sofreram adaptações para um melhor aproveitamento dos alunos. As atividades para ambos os softwares consistiam em aulas expositivas e, posterior a essa etapa, os participantes foram convidados a realizarem atividades práticas pertinentes aos conteúdos trabalhados.

\subsubsection{Dosvox}

Após apresentar os recursos básicos de informática, iniciou-se o ensino da ferramenta Dosvox. Com a intenção de familiarizar os participantes com o software, o primeiro contato foi com o teste do teclado. Esse teste é de grande importância, pois somente após o reconhecimento do teclado, o usuário poderá utilizar o programa com maior facilidade e agilidade, uma vez que o uso do mouse para o deficiente visual está descartado. Durante o teste do teclado, os alunos foram convidados a explorá-lo com relação à localização das letras, caracteres, entre outras funcionalidades.

Depois de adquirirem habilidades práticas no manuseio do teclado, foram ministradas aulas sobre os conteúdos dispostos na Tabela 2.

Tabela 2. Componente curricular do software Dosvox

\begin{tabular}{|c|l|}
\hline Conteúdos & \multicolumn{1}{c|}{ Descrição } \\
\hline Síntese de voz & $\begin{array}{l}\text { Realização de comunicação entre o Dosvox e o } \\
\text { deficiente visual. }\end{array}$ \\
\hline $\begin{array}{c}\text { Editor, leitor e } \\
\text { impressor/formatador de }\end{array}$ & $\begin{array}{l}\text { Práticas para o aluno trabalhar com eficácia na } \\
\text { criação, formatação e edição de textos, bem como }\end{array}$ \\
\hline
\end{tabular}




\begin{tabular}{|c|l|}
\hline textos & $\begin{array}{l}\text { a utilização de recursos para a formatação de } \\
\text { fontes, parágrafos e páginas, entre outros. }\end{array}$ \\
\hline Recursos multimídia & $\begin{array}{l}\text { Utilização de diversos programas de uso geral } \\
\text { como: Midiavox, jogos, gravador de som e entre } \\
\text { outros. }\end{array}$ \\
\hline Internet & $\begin{array}{l}\text { Utilização de programas sonoros para acesso ao } \\
\text { Correio Eletrônico do Dosvox. }\end{array}$ \\
\hline
\end{tabular}

Durante as atividades práticas iniciais, foi observado que os participantes não conseguiram realizar as atividades com eficiência. Essa dificuldade ficou evidente durante as aulas práticas sobre o Edivox - editor de texto. Esse fato ocorreu devido ao Dosvox recorrer a utilização de comandos para o acesso aos seus aplicativos (ou subprogramas). Isto provocou nos usuários um desconforto e demora para conseguir encontrar a ação desejada na lista de opções. Com a intenção de ilustrar uma dessas atividades, a Figura 1 apresenta uma lista de opções do Edivox com as suas ferramentas para formatação de textos.
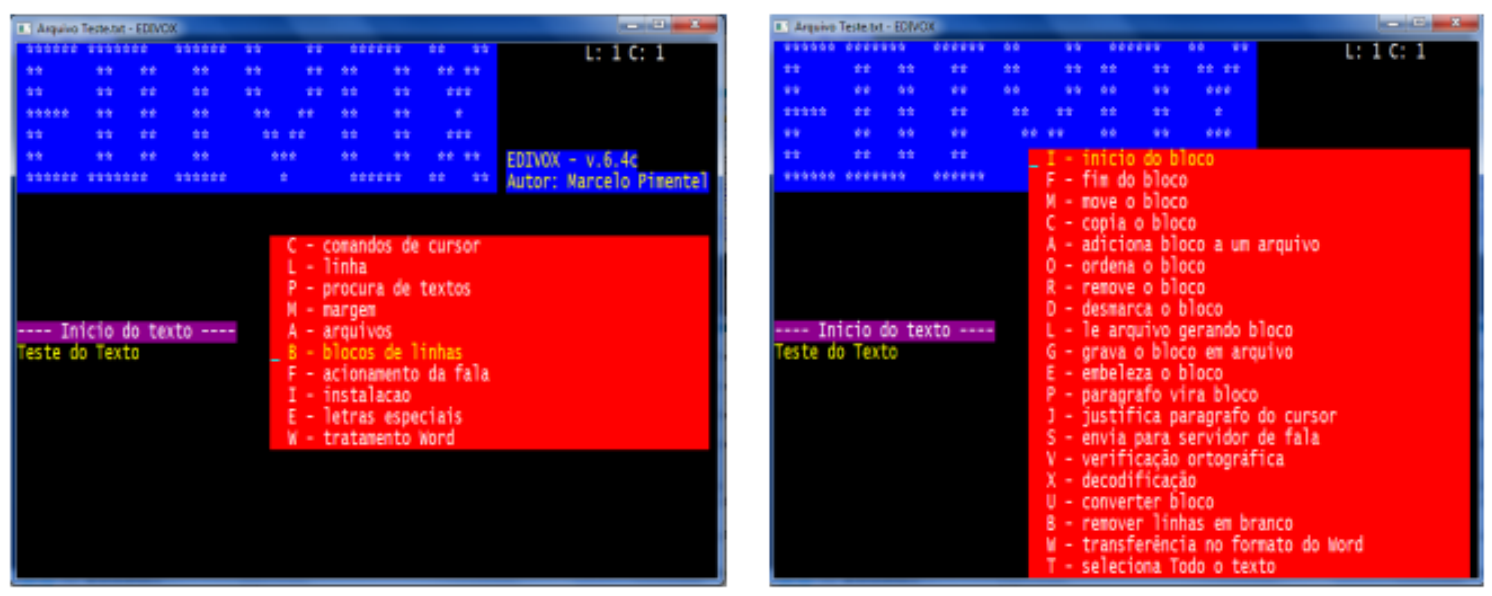

Figura 1. Menu do Edivox com lista de comandos do Dosvox.

Após serem ministrados os conteúdos sobre edição, leitura, impressão de textos e manipulação de arquivos, os alunos tiveram momentos de descontração e interação com o uso dos Jogos Educacionais (Jogo da Forca, Jogo da memoria, paciência e Contavox) presentes no próprio Dosvox. Essa experiência, a qual pode ser vista na Figura 2, foi de grande utilidade para os participantes devido ao fato desses jogos não terem somente como objetivo o entretenimento, mas também facilitar a aprendizagem do ambiente. À medida que o usuário joga, ele está ao mesmo tempo aperfeiçoando sua interação por meio do teclado e com o sistema de um modo geral. 

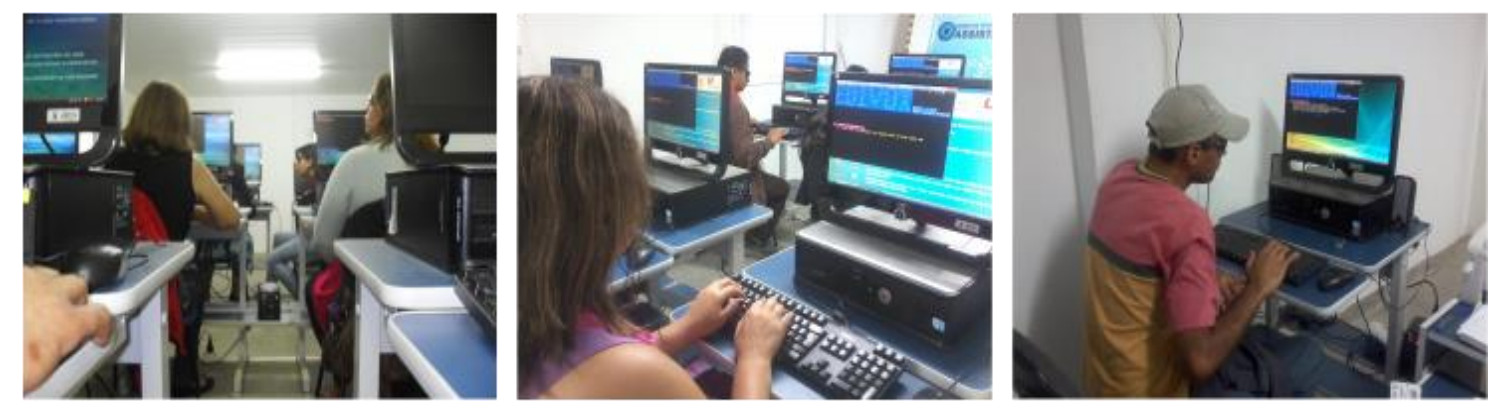

Figura 2. Participantes do curso utilizando o Dosvox

Em seguida, foram ministradas aulas sobre o Cartavox. Este consiste de um programa de acesso à internet que permite o envio e recebimento de e-mails. Inicialmente foram apresentadas as funcionalidades do Cartavox e as tarefas a serem realizadas, sendo elas:

a) Configurar o Cartavox com seu e-mail pessoal;

b) Enviar e-mail (esta função apenas prepara o e-mail não enviado);

c) Receber e-mails no Cartavox;

d) Folhear e-mails já recebidos;

e) Transmitir e-mails escritos (envia o e-mail preparado anteriormente);

f) Enviar e-mail com arquivo anexo.

Durante a execução das tarefas, foi observado que os participantes apresentaram dificuldades com a configuração de e-mail do Cartavox por requerer habilidade e conhecimento de termos técnicos como, por exemplo: servidor, SMTP, provedor etc. No entanto, na realização das atividades de enviar, receber, folhear e transmitir e-mails, os participantes não apresentaram dificuldades, o fluxo delas transcorreu normalmente.

\subsubsection{NVDA}

No curso de formação, o software NVDA foi utilizado apenas para auxiliar o acesso à internet. O componente curricular para o ensino do NVDA pode ser observado na Tabela 3.

Tabela 3. Componente curricular do software NVDA

\begin{tabular}{|c|l|}
\hline Conteúdos & \multicolumn{1}{|c|}{ Descrição } \\
\hline Configurações do Leitor & $\begin{array}{l}\text { Teclas de atalhos para iniciar e parar a leitura da tela, } \\
\text { configuração do volume, da velocidade, tipo e tom } \\
\text { da voz do sintetizador. }\end{array}$ \\
\hline Utilização do NVDA & Lista de Teclas de Comando do NVDA. \\
\hline Acesso a Web & $\begin{array}{l}\text { Acesso a páginas Web e ao serviço de e-mail } \\
\text { (Gmail). }\end{array}$ \\
\hline
\end{tabular}

Primeiramente, foram apresentadas as configurações iniciais do NVDA para que os participantes pudessem configurá-lo de acordo com suas preferências quanto ao tipo, velocidade e tom da voz do leitor. Após essa fase, foi repassada aos participantes a lista de teclas de comando (combinação de teclas) do NVDA. Isso se faz necessário visto que 
ao acionar esses comandos, o NVDA irá anunciar todas as teclas que são ativadas por meio do teclado para que assim os deficientes visuais possam acessar e interagir com o sistema operacional Windows juntamente com outros aplicativos.

Para as aulas teóricas sobre acesso a Web, inicialmente foi utilizada uma interface tátil, em alto relevo, como pode ser observada na Figura 3. O sentido do tato ocorre através da percepção e interpretação por meio da exploração sensorial. Uma representação tátil deve apresentar um conjunto de texturas, diferentes superfícies e consistências a fim de promover o reconhecimento da estrutura do objeto e da relação das partes com o todo (GRIFFIN; GERBER, 2013). Nessa representação tátil, os participantes puderam perceber pela primeira vez a disposição dos principais componentes da interface Web a ser acessada, tais como: botão, menus, barra de rolagem, disposição do conteúdo, caixa de busca, localização da logomarca e rodapé do site. Esta atividade provocou nos participantes um sentimento de prazer por ter sob controle os recursos que pretendiam utilizar em cada página.
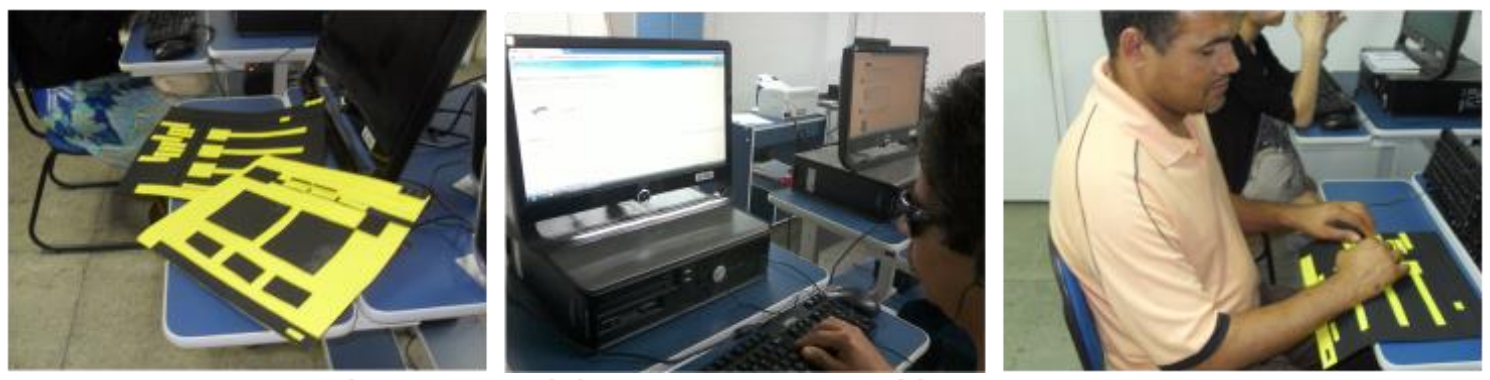

Figura 3. Participantes do curso utilizando o NVDA

Nas aulas práticas, os participantes foram convidados a acessar as páginas da Web de seu interesse pessoal com o objetivo de motivá-los a buscar novas fontes de conhecimento e informação além dos materiais impressos em Braile (sistema de escrita e leitura dos cegos). Para possibilitar aos deficientes visuais ampliar sua comunicação através do meio digital, foi proposto que os participantes criassem uma conta de e-mail no servidor Gmail da Google. O Gmail foi escolhido por fornecer acessibilidade aos usuários deficientes visuais. Após o cadastro, os participantes foram convidados a realizar as seguintes tarefas: a) acesso do usuário ao e-mail (identificação por meio do nome de usuário e senha), b) enviar um e-mail para um colega da própria turma e c) ler e-mails da caixa de entrada.

Com esta simulação, notou-se que a navegação em uma página Web através da utilização prévia de uma representação tátil, permitiu aos participantes perceberem mais precisamente onde os recursos do e-mail estavam organizados. Portanto, comprovou-se que a interação dos deficientes visuais com representações táteis auxilia na construção de modelos mentais mais significativos (PREECE, 2005).

\section{Considerações Finais}

Este artigo teve a intenção de demonstrar a experiência do ensino de informática para deficientes visuais do município de Garanhuns, onde contou com o apoio da Associação dos Deficientes Visuais do Agreste Meridional de Pernambuco (ADVAMPE) da Secretaria de Desenvolvimento Econômico deste município e da Empresa Júnior TEC Jr. Após a caracterização do perfil do público-alvo, o curso de formação foi desenvolvido e conduzido de acordo com as necessidades de cada aluno participante. 
De uma maneira geral, este projeto propôs uma reflexão a respeito do uso das Tecnologias Assistivas, visto que estas facilitam o acesso dos deficientes visuais ao computador, garantindo-lhes maior autonomia, oportunizando o acesso a ambientes digitais favorecendo assim sua inclusão social e digital. Portanto, pode-se concluir que o objetivo deste trabalho caracterizado pela promoção de um curso de formação sobre o uso das Tecnologias Assistivas como ferramentas facilitadoras do processo de inclusão social e digital foi alcançado com sucesso pela perspectiva dos alunos e dos tutores.

De acordo com o relato dos participantes, o curso promoveu uma mudança de perspectiva sobre a importância do uso do computador. A partir do conhecimento adquirido, eles argumentaram que irão poder pesquisar sobre informações de seus interesses, estudar de forma mais independente e participar de redes sociais, algo que eles ouvem falar bastante. Este curso foi reconhecido tanto pela internamente na Universidade de Pernambuco, quanto regionalmente pelo seu sucesso, como pode ser observado nas publicações no Jornal Extra ${ }^{4}$ e no blog de Ronaldo César ${ }^{5}$, importante blogueiro do município de Garanhuns.

Espera-se que este trabalho possa servir de exemplo para outras iniciativas semelhantes e que alunos dos cursos de Licenciatura em Computação se conscientizem para promover projetos pedagógicos destinados ao desenvolvimento de estratégias de ensino e aprendizagem que beneficiem deficientes visuais no uso adequado de recursos tecnológicos. Como ação futura, pretende-se realizar cursos de formação voltados para o ensino de Computação com alunos deficientes visuais em Garanhuns e região vizinha com o objetivo de estimular o pensamento computacional com a aplicação de técnicas para a resolução de problemas contextuais desse perfil de público.

\section{Referências}

Borges, Antonio José. (2012) "Dosvox: Um Novo Acesso dos Cegos à Cultura e ao Trabalho", Disponível em: < http://www.ibc.gov.br/?itemid=100>, Acesso em Julho de 2013.

Dosvox. (2012) “Projeto Dosvox", Disponível em: <http://intervox.nce.ufrj.br/Dosvox>, Acesso em Junho de 2012.

Ferreira, S., Bacellar L. and Nunes, Ricardo Rodrigues. (2011) “e-usabilidade”. Rio de Janeiro, RJ: LTC.

Glat, Rosana. (2007) “Educação Inclusiva: cultura e cotidiano escolar.” Rio de Janeiro RJ: Letras.

Griffin, H. C. and Gerber, P. J. (2012) "Desenvolvimento Tátil e suas Implicações na Educação de Crianças Cegas", Disponível em: http://www.ibc.gov.br/?itemid=100, Acesso em Julho de 2013.

IBGE, Censo demográfico. (2010) "Pessoas Deficientes", Disponível em: < http://www.ibge.gov.br/home/estatistica/populacao/censo2010/resultados_preliminar

\footnotetext{
4 Vide notícia: http://www.jornalextra.com.br/portal/blog/2012/06/18/projeto-de-inclusao-digitalbeneficia-deficientes-visuais-no-agreste-pernambucano/

5 Vide notícia: http://blogdoronaldocesar.blogspot.com.br/2012/05/alunos-de-computacao-da-upecriam.html
} 
es_amostra/default_resultados_preliminares_amostra.shtm>, Acesso em Junho de 2012.

Jornal Extra (2012) "Projeto de inclusão digital beneficia deficientes visuais no Agreste pernambucano", Disponível em: < http://www.jornalextra.com.br/portal/blog/2012/06/18/projeto-de-inclusao-digitalbeneficia-deficientes-visuais-no-agreste-pernambucano/>, Acesso em Agosto de 2013.

NVDA. (2012) "Manual NVDA: Leitor de Tela: Guia do Usuário”. Disponível em: < http://acessibilidadelegal.com/33-manual-nvda.php>, Acesso em Junho de 2013.

Pansanato, L. T. E., Silva, C. E. and Rodrigues, L. (2012) "Uma Experiência de Inclusão de Estudante Cego na Educação Superior em Computação”, XX Workshop sobre Educação em Computação.

Preece, J. J., Rogers, Y. and Sharp, H. (2005) "Design de interação: além da interação homem-computador". Hoboken, NJ: BOOKMAN COMPANHIA.

Cesar, Ronaldo (2012) "Alunos de computação da UPE criam projeto social de informática para deficientes visuais", Disponível em: < http://blogdoronaldocesar.blogspot.com.br/2012/05/alunos-de-computacao-da-upecriam.html>, Acesso em Agosto de 2013.

Santos C. P., Ellwanger C., Stangherlin V. and Kilian Y. (2012) Projeto Infoacesso Informática para portadores de Deficiência Visual. Revista Vivências. Vol.8, N.14: p.200-209, Maio/2012.

Souza, A. P. (2004) "Acessibilidade de Deficientes Visuais aos Ambientes Digitais/Virtuais", Dissertação de Mestrado, Universidade Federal do Rio Grande do Sul, Porto Alegre. 